\title{
The Effect of Botulinum Toxin on the Platysma Muscle
}

\author{
Bhertha M. Tamura
}

Published online: 18 April 2012

(C) Springer Science+Business Media, LLC 2012

\begin{abstract}
Even before physicians began to inject botulinum toxin A (BTXA) for the treatment of facial dynamic lines, we were already aware of the possibility of treating the platysma band and the transversal lines of the neck. There were few doctors claiming good results, but there were still questions about why, when, how, and in whom how to inject BTXA, as well as questions about the clinical results and complications of course. Now that we have being treating the lines of the neck through the platysmal bands for some time, with great emphasis at the contouring of the jaw line for the global facial rejuvenation, we can discuss treatment of the platysma muscle and analyze its dynamics, identification, and limits, and also have a better idea of how to manage the injection of BTXA in this area with good results. This article analyzes the pattern of a series of platysma muscle treatment to determine if there is a model that could be followed for the contouring of the face and neck by comparing the current knowledge about the behavior, position, and results of the BTXA injection sites based on the initial idea of treating the platysmal bands and the transversal cervical lines of the neck. We randomly selected 40 patients from our private clinic treated between 2006 and 2011 who had have botulinum toxin injections for the recontouring of the face to analyze if there was a pattern of injection to follow as a model for the treatment of the platysma. They were all female, as there were no males that
\end{abstract}

B. M. Tamura $(\bowtie)$

Rua Ituxi, 58 apto 603, Bairro Mirandópolis,

São Paulo, SP, Brazil CEP 04055-020

e-mail: bhertha.tamura@uol.com.br

B. M. Tamura

Lexbios Medical S/S Ltda,

São Paulo, Brazil had had any treatment for this indication in our files. These patients had received at least two treatments, resulting in a total of 90 treatments for the neck during 15 months, with the total dose injected per session varying from 10 to 70 units. We have made a retrospective evaluation of the preand post-treatment pictures, checking the effective clinical results after the treatment and making sure that the patient was included in the discussion. There was not any exact model of points, distribution, quantity, and constant pattern for the treatment of the platysma muscle for the contouring of the face. There were differences between the patients and in the same patient (e.g., different pattern from the right side and the left side). The clear knowledge of the muscular distribution through its dynamic action to work as a mimetic muscle that might change the facial lower contour is the key aspect for this treatment.

Keywords botulinum toxin - platysma $\cdot$ band $\cdot$ rejuvenation of the neck $\cdot$ cervical platysmal bands

\section{Introduction}

Even before physicians began to inject botulinum toxin A (BTXA) for the treatment of facial dynamics lines, we were already aware of the possibility of treating the platysma band and the transversal lines of the neck. There were few a doctors claiming good results, but there were still questions about why, when, how, and in whom how to inject BTXA, as well as questions about the clinical results and complications of course. Now that we have being treating the lines of the neck through the platysmal bands for some time, with great emphasis at the contouring of the jaw line for the global facial rejuvenation, we can discuss treatment of the platysma muscle and analyze its dynamics, identification, and limits, and also 
have a better idea of how to manage the injection of BTXA in this area with good results.

The platysma is currently described as a muscle that distends the skin of the neck and pulls it downward and laterally, lowering the corners of the lower lips by pulling down the lateral corner of the mouth angle and acting in synergy with the depressor anguli oris (DAO) (Fig. 1). Platysmal posterior fibers continue superiorly to join the superficial musculoaponeurotic system (SMAS) of the face and all the inferior facial muscles act together in a complex relationship including the deep lower lip depressor, the mentalis, and the DAO muscles (making the famous "M" shape at the chin area. Platysma originates from the sternoclavicular joint, clavicular bone, and the scapular acromion, inserting itself at the inferior border of the mandible and few fibers at the mouth angle (although sometimes fibers can be seen in the superior portion of the chest) (Fig. 2). It is assumed that the anterior portion is very strong and that it directs to the anterior region of the chin toward the lips and finally ends at the modiolus. In some individuals it could also se extend laterally until the jaw angle (Fig. 1). There are variations in the way the platysma band joins together; and sometimes they might not join except at the chin (Fig. 2), they might have no decussations, they may be completely separated in about one third of the patients, and in two thirds of the cases they join at the anterosuperior neck. They can crisscross or the decussation might cover one half or more the distance from the chin to the hyoid bone. One third of the time, the decussations are absent or a complete separation occurs, but all these anatomical variations can be completely different from one side of the neck to the other in the same patient [1-4].

Kane [5] introduced the platysmal band BTXA treatment in 1999, but 1 year earlier Brandt and Bellman [6] has already published data about the use of the BTXA for cosmetic treatment of the neck. Park et al. [7] and Brandt and Boker [8] had described the treatment of the lower face contour. In

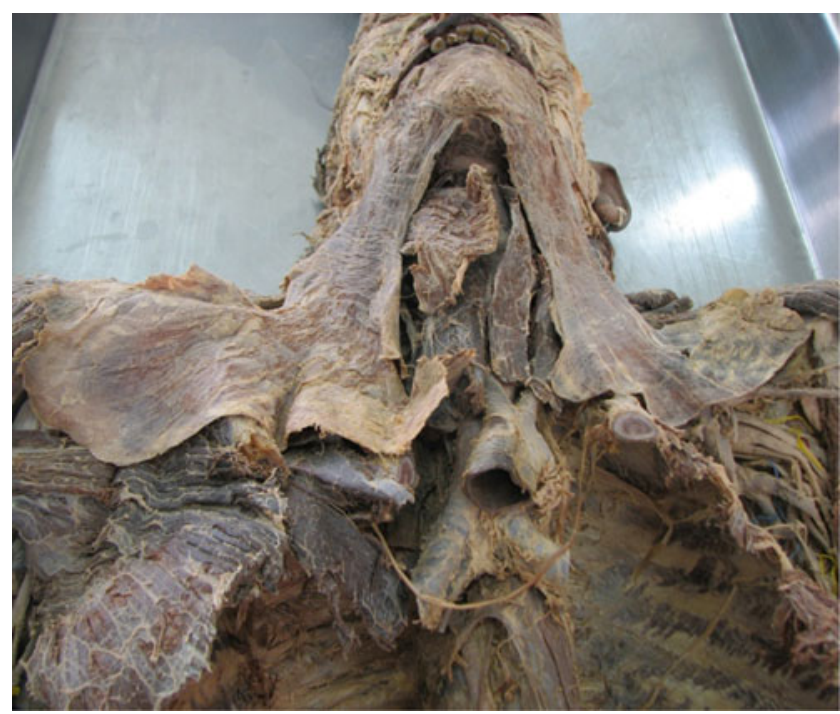

Fig. 2 It is assumed that the anterior portion of the platysma is very strong and that it directs to the anterior region of the chin toward the lips and finally ends at the modiolus. In some individuals it could also be extend laterally until the jaw angle, as show in this cadaver dissection

2007, Levy [9] injected BTXA along the inferior border of the mandible and at the platysma band that pulled the skin downward and named this treatment the "Nefertiti lift." Following this article, we saw that the results were not constant and returned to our original technique to treat the skin lateral to the mouth corner (the "bulldog" ageing problem) (Fig. 3). In 2007, we [10] published that BTXA injection of $5 \mathrm{U}$ could result in a good lower contour of the face (Fig. 4).

\section{Methods}

We randomly selected 40 patients from among 203 patients from our private clinic treated between 2006 and 2011 who had been treated with botulinum toxin (onabotulinum toxin

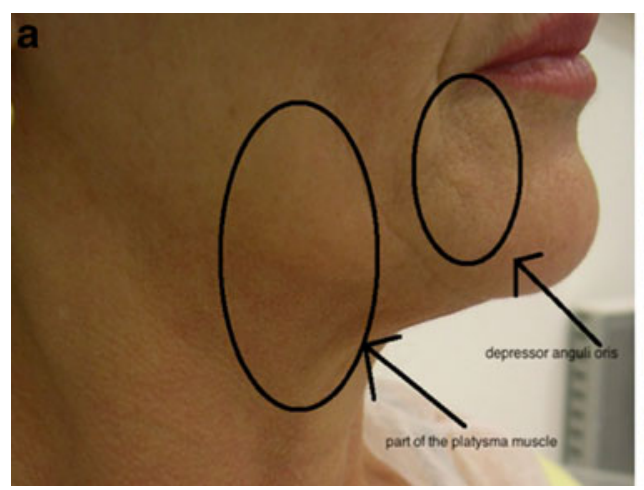

Fig. 1 a Platysma distends the skin of the neck and pulls it downward and laterally, lowering the corners of the lower lips by contributing to pulling down the lateral corner of the mouth angle and acting in synergy. $\mathbf{b}$ demonstration of the depressor anguli oris and platysma

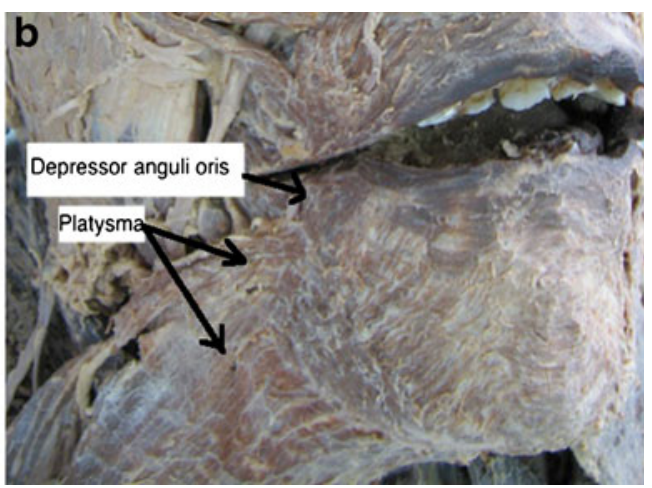

muscle in dissection of cadaver. It is assumed that the anterior portion of the platysma muscle is very strong and that it directs to the anterior region of the chin toward the lips and finally ends at the modiolus. In some individuals it could also se extend laterally until the jaw angle 
Fig. 3 The skin lateral to the mouth corner that we have termed the "bulldog" aging problem

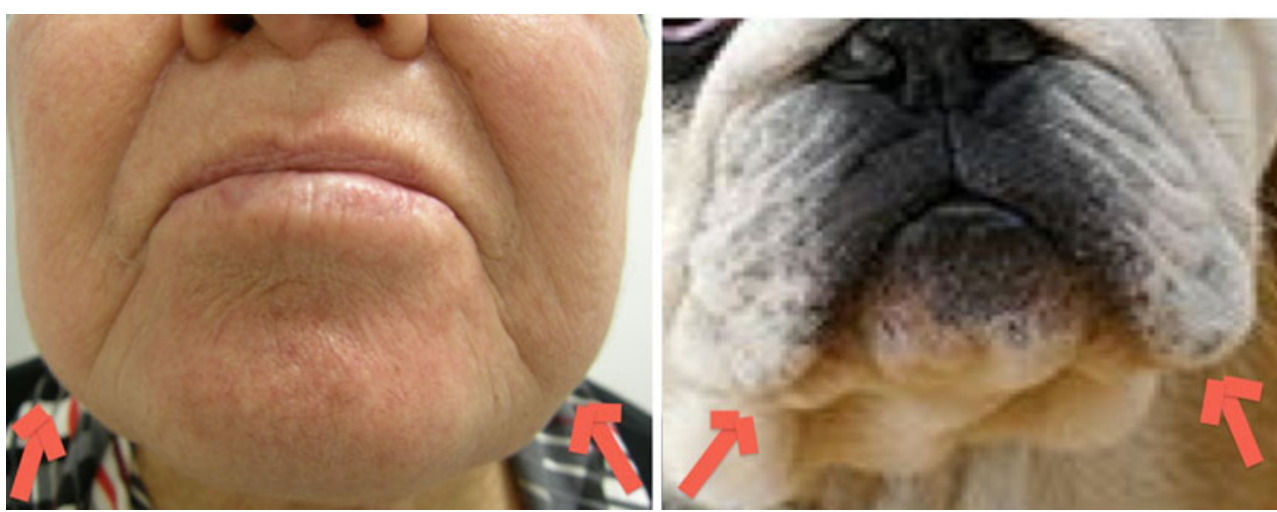

A) $100 \mathrm{U}$ diluted in $1 \mathrm{~mL}$ of saline specifically for jaw line re-contouring to analyze if there was a pattern of injection to follow as a model for the treatment of the platysma. The patients were all female, between 38 and 62 years of age (median, 53 years) with a skin type between I and III (four patients were skin type IV). There were no men that had had any treatment for this indication or any complaints about their neck in our files. We made a 15-month retrospective evaluation of the pre- and post-treatment pictures, checking the clinical results that were achieved after the treatment to be sure that the patient was included in this discussion. They all had received follow-up and their pictures had been taken after 14 days or up to 20 days.

In our clinic, all patients have a pre-procedure file and a signed consent before any cosmetic treatment. There is also a questionnaire in which we strongly advise that BTXA treatment not be performed in pregnant and breast feeding women, if they have a personal or familial history of myasthenia gravis, Eaton-Lambert syndrome, any kind of infection, association of other drugs that can compete with BTXA, or psychological disorders. We also recommend that actors, singers, politicians, and lawyers should avoid extensive treatment in order to keep the facial mimetic.

We always have had as a priority in treating the individual's anatomy to choose the amount of units in determining which muscle or portion of the muscle to treat, so all cervical platysma muscle are evaluated with dynamic movements. As a routine at our everyday practice, the patients were always checked looking forward with the neck straight and relaxed so that we could be sure they needed BT for the platysma. Once a decision to treat the contour of the face (specially platysma) was made, we would asked them to force the depressor angulus oris muscle downward and when they could not do it well, they were asked to vocalize a continuous and forced "e" sound to keep the platysma muscle contracted. The key objective was relaxing the portion of the platysma that pulled downward the lateral portion of the jaw area lateral to the corner of the mouth (i.e., the "bulldog" shape) (Fig. 3).

Once the platysma area that pulls the skin was identified, the role of effective muscular area was delimited, including the bands' shape, with a proper skin marker pencil. The strongest and most active portion of the muscle were identified and marked, and at this point it would receive between 3 and $5 \mathrm{U}$ (maximum per point) and the points lateral to this main point received at least $1 \mathrm{U}$ less than the first and the others received $2 \mathrm{U}$ each. The injection points were always analyzed individually, and in the same patient sometimes one side can be different than the other. The distance between the injections sites was always kept as $1.5 \mathrm{~cm}$. Usually, there were three main injection points right under the mandible border and the minimal units injected in some patients was 1 unit at the weakest parts of the bands down the neck, near the clavicle. The injections of the lateral aspect of the neck were performed intramuscularly and the injections at the central (medium) part of the neck were always intradermal.
Fig. 4 Before (left panel) and after (right panel) BTXA injection of $5 \mathrm{U}$ for good lower contour of the face
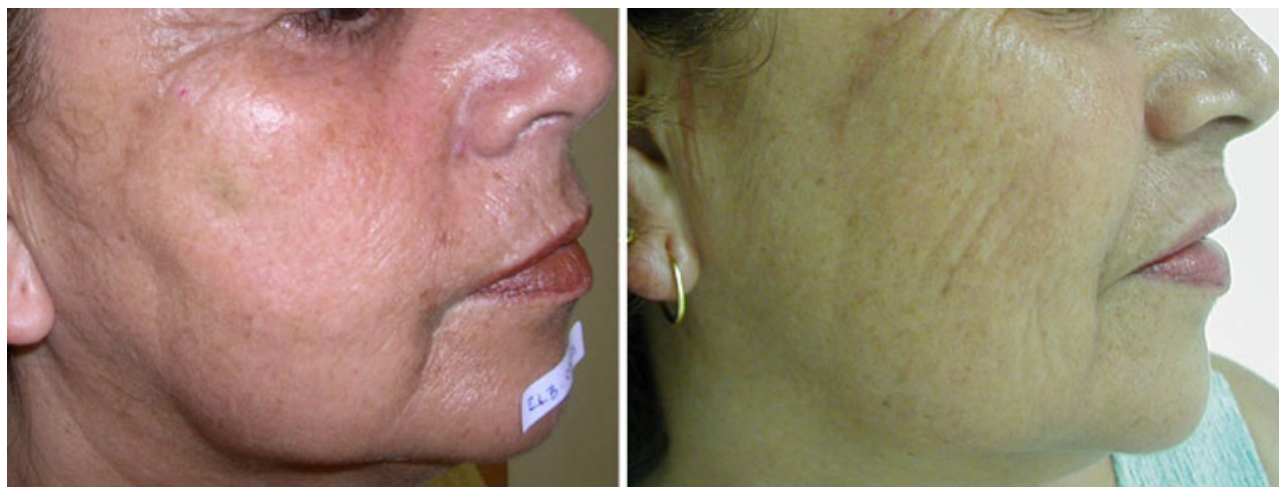
Fig. 5 An example of the difference between the right side (left panel) and left side (right panel) of a patient, with the dots indicating the points of BTX-A injections. The right side recieved a total dose of $22 \mathrm{U}$ and the left side received a dose of $20 \mathrm{U}$
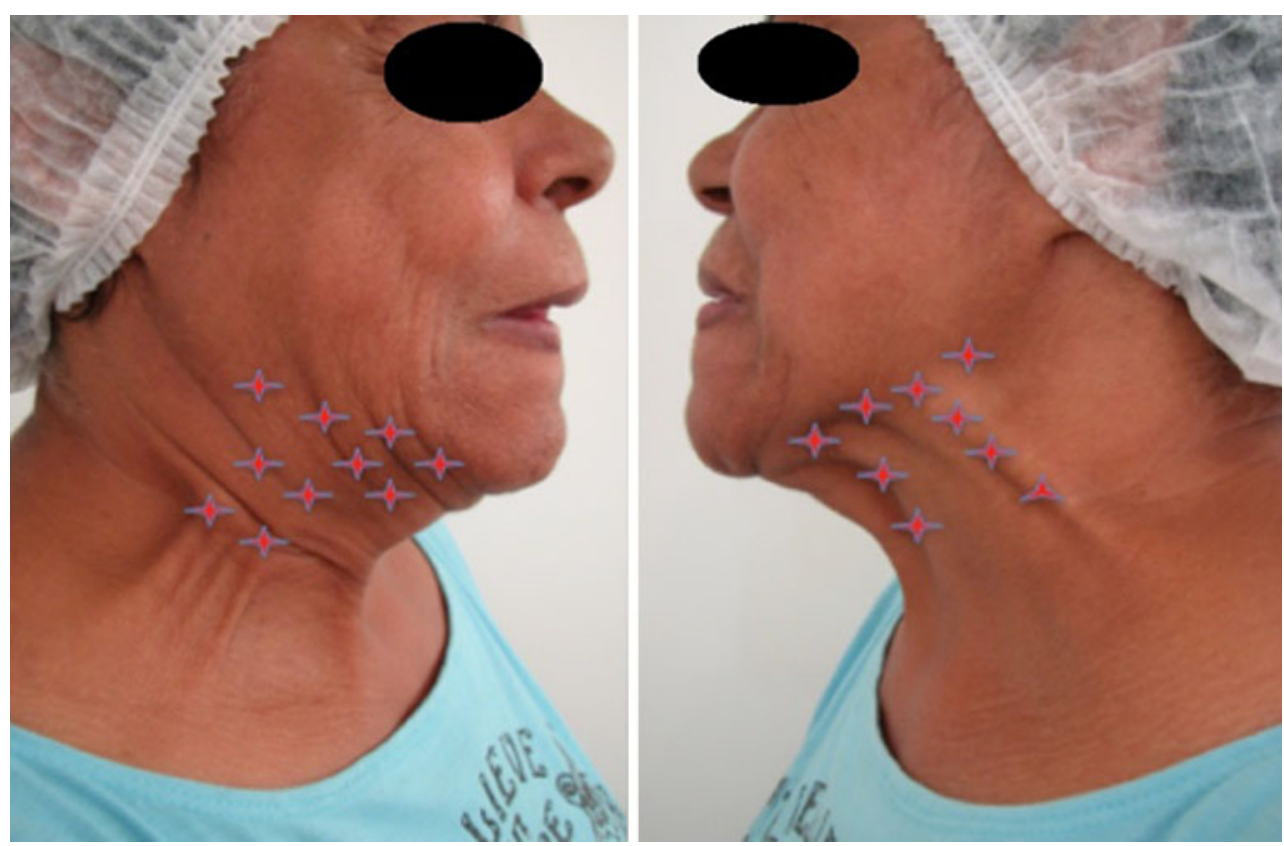

\section{Results}

The 40 patients received at least two treatments, resulting in a total of 90 treatments for the neck during 15 months, with the total dose injected per session varying from 10 to $70 \mathrm{U}$ (median dose, 36 units). The smallest amount injected per patient was $10 \mathrm{U} ; 5 \mathrm{U}$ on each side of the most powerful muscular contraction point of the platysma, and $70 \mathrm{U}$ was the maximum dosage (one patient).

The pattern was so different from one patient to the other that we could not establish an adequate classification of the clinical aspect of the platysma. Even in the same patient, sometimes the lateral aspect of the oral anguli and the skin ("bulldog") could be pulled by the anterior band, the posterior band, by the platysma between the anterior and posterior band, or by two portions of the muscle. Figure 5 is an example of the difference between the right and left side at same patient, and Figs. 6, 7 and 8 show the patient before and after treatment with a nice contouring of the jaw line.

Although the majority of the patients had improvement with only a single treatment at their return check up, we also noticed that if the patient had strong bands and that if one of them had not been treated at the first appointment it needed to be injected in the return check as an extra complimentary treatment at 14 to 20 days of evaluation (three patients).

Fig. 6 Before (left panel) and after (right panel) photos of a patient, with a nice contouring of the jaw line
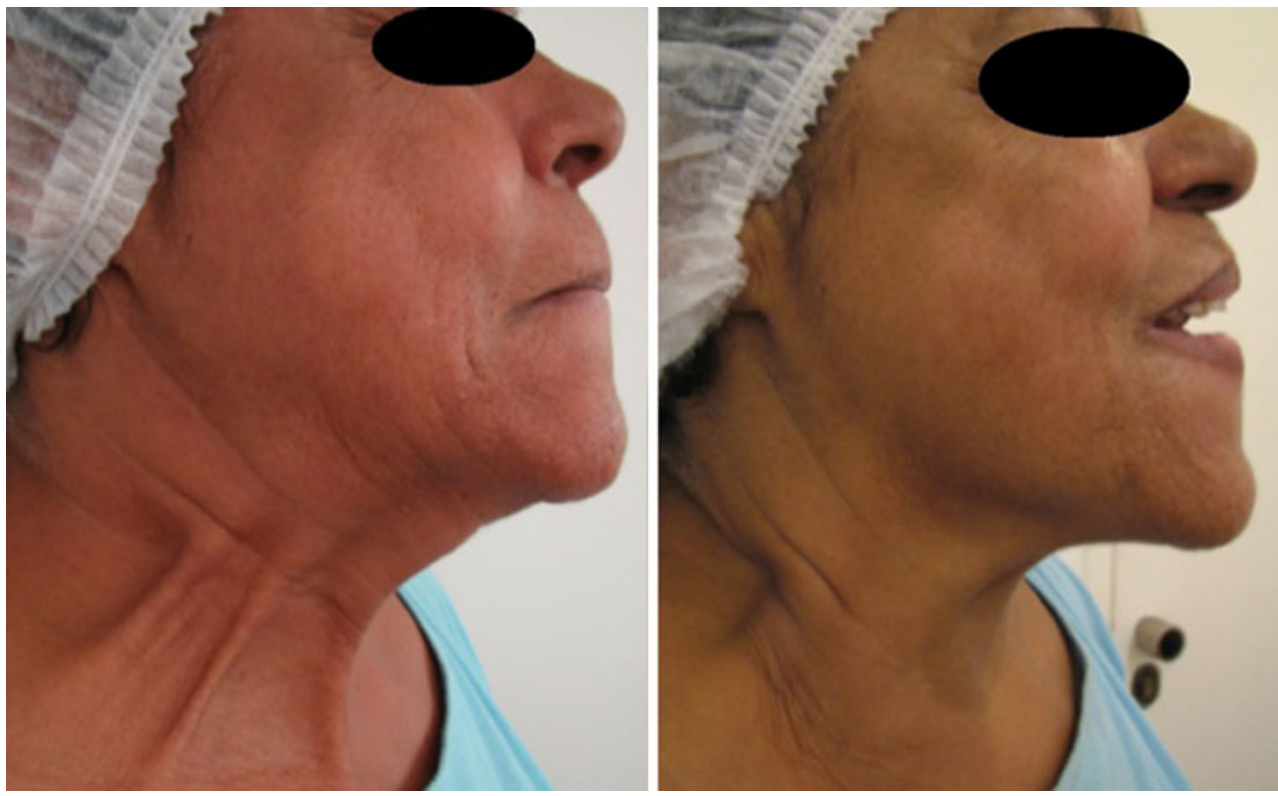
Fig. 7 Before (left panel) and after (right panel) photos of a patient, with a nice contouring of the jaw line

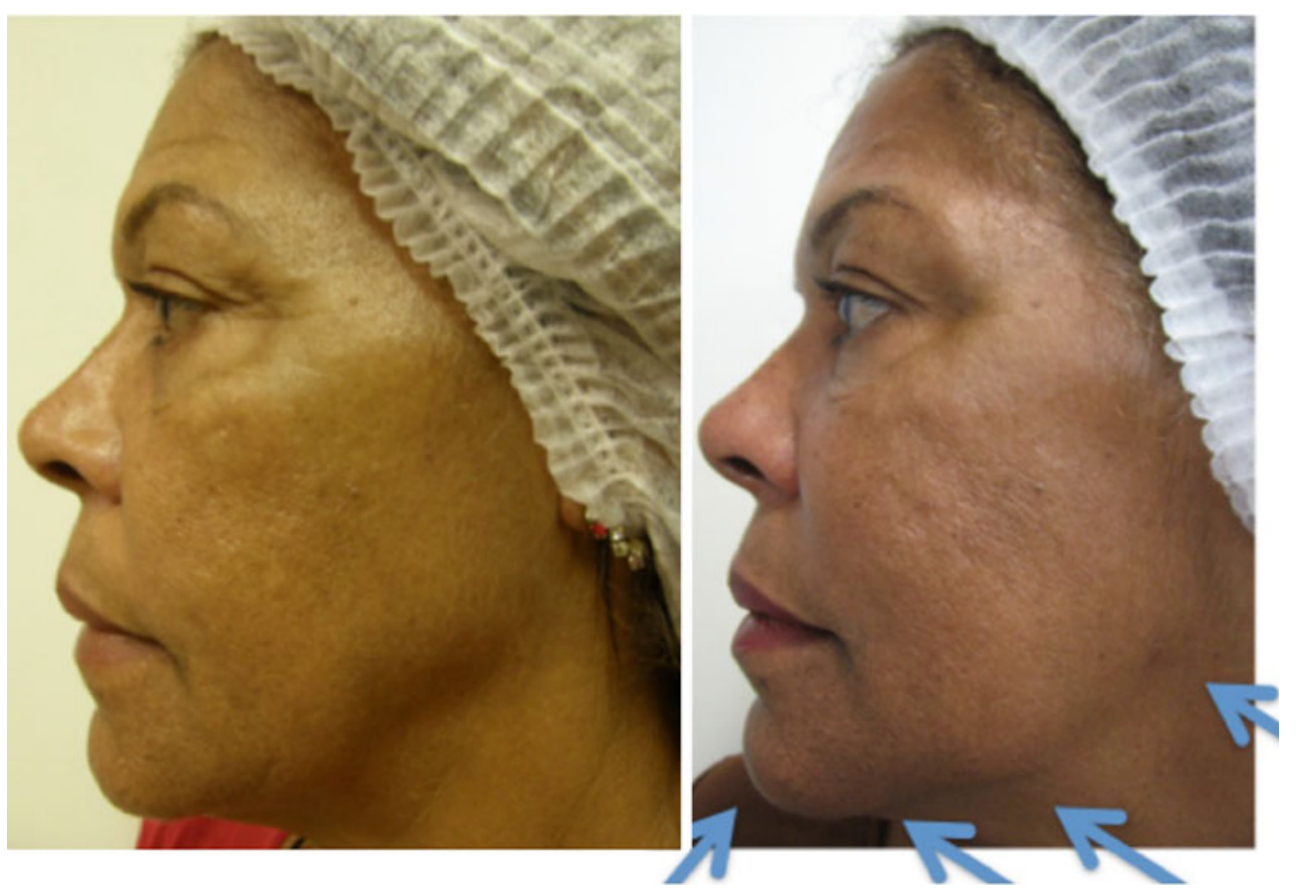

Four patients that had received between 50 and $70 \mathrm{U}$ reported impairment in their ability pull their head from the pillow in the mornings (compromise "sit ups") or when they had their hair washed in hairdresser's special couches, sometimes needing some self-hand help to pull the head in the first 4 weeks.

After having treated patients in the platysma muscle, we realized that the transversal bands had a considerable improvement, but this was not the objective of our study.

\section{Discussion}

When we began our practice we treated only the portion of the platysma that clearly pulled the skin of the lateral part of the oral angle. Later on, in trying to follow a pattern of treatment like Nefertiti's technique, we found we were not successful in at least half of the patients, leading us to analyze anatomically what was really happening and why we had so many failures in patients for the treatment of the lower face contouring. The answer had a simple explanation: individual anatomic variations.

Every patient is unique, so the physician must detail all the bands, border, and the most important part of the platysma (which will receive a bigger amount of units) to have success. We should remember that there are patients that have not decussation that might have the "bulldog" pulled by the anterior band, medium, posterior, or association of bands through the strength of the DAO pulling even more on the de anguli oris. Not only is the contouring of the lower face important [11], but it is also must be remembered that the platysma band can be located along the claviculae, inferior to it, or even until the upper part of the breast.

The anatomical distribution of the platysma is different from one patient to the other, and can be different even in the same patient. The patients must be evaluated carefully,

Fig. 8 Before (left panel) and after (right panel) photos of a patients, with a nice contouring of the jaw line; notice the improvement of the transversal line. Total dose used for this side of the neck was $16 \mathrm{U}$
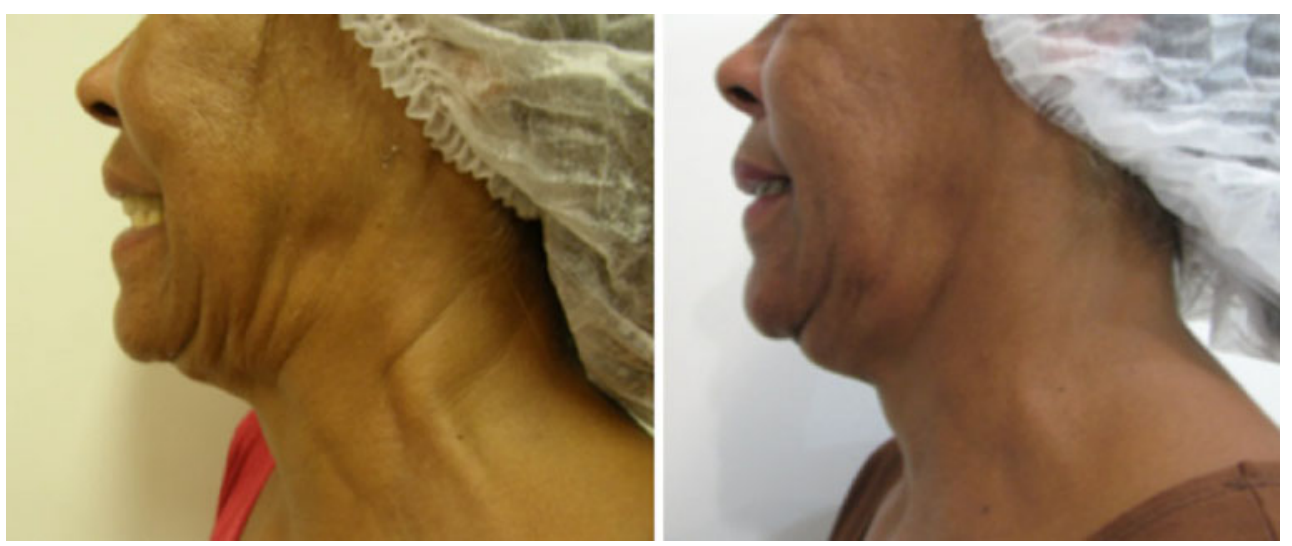
with consideration being given to each side of the neck, in particular to the dynamic action of the muscle, identifying the bands $[12,13,14 \cdot \bullet]$, the transversal lines, and the portion of the platysma that is actually pulling down the "bulldog" skin of the face. All the strong bands should be relaxed with BTXA, with smaller amounts but always enough so that they will not need a touch up.

Injecting more than $50 \mathrm{U}$ might be of risk considering the neck weakness. Some of the patients reporting dysphagia could have it due to the relaxation of the mylohyoid and stylohyoid muscle, and in order to prevent that we have being injecting BTXA at the central anterior (medium) part of the neck very superficially, in the intradermal level if possible. We had no patients with dysphagia, but ecchymosis in one or more sites of injection was relatively frequent (20 patients).

We avoid as much as possible injecting more than 30 to $50 \mathrm{U}$ for the treatment of the platysma, and if it is correct, the definition of the procedure should actually be the treatment of the neck lines, the jaw line, and the "bulldog skin and not only the platysmal band.

The improvement of the transversal lines of the neck leads to another question: whether or not the patient will need an asymmetric amount of BTXA for the treatment of the neck. We suggest that if the difference is great, we should inject small amounts of BTXA just below or above the lines $(1-2 \mathrm{U})$ of the side that will receive fewer units.

Although this study considers only the treatment of the platysma muscle, we need to discuss the association of fillers $[15 \bullet 16 \bullet 17 \bullet, 18 \bullet]$, the treatment of the DAO, and the treatment of the mental muscle to improve the results. The flaccidity of the skin through the submental fat could lead to an unsatisfactory outcome [19•, 20••]. Some patients that do have hypognatism can experience some improvement.

\section{Conclusions}

There was not an exact distribution of the points, quantity, and a constant pattern for the treatment of the platysma muscle for the contouring of the face. There were differences between the patients and in the same patient, and different patterns from the right side and the left side. The clear knowledge of the muscular distribution in its dynamical contraction to act as a mimetic muscle that influences the facial lower contour is the key aspect for the treatment of this muscle for a real clinical improvement.

Disclosure No conflicts of interest relevant to this article were reported.

\section{References}

Papers of particular interest, published recently,

have been highlighted as:

- Of importance

•- Of major importance

1. Tamura BM. Anatomia da face aplicada aos preenchedores e à toxina botulínica - parte I. Surgical \& cosmetic dermatology. Surg Cosmet Dermatol. 2010;2(3):195-204.

2. Altruda Filho L, Cândido PL, Larosa PRR, Cardoso EA. Anatomia topográfica da cabeça e do pescoço. 1st ed. Barueri: Manole; 2005.

3. Gardner E, Gray DJ, O'Rahilly R. Anatomia. 4th ed. Rio de Janeiro: Guanabara Koogan; 1978.

4. Sobotta J, Becher H. Atlas de Anatomia Humana, vol 1 a 3. 17th ed. Rio de Janeiro: Guanabara Koogan; 1977.

5. Kane MA. Nonsurgical treatment of platysma bands with injection of botulinum toxin A. Plast Reconstr Surg. 1999;103:656-63.

6. Brandt FS, Bellman B. Cosmetic use of botulinum A exotoxin for the aging neck. Dermatol Surg. 1998;24(11):1232-4.

7. Park MY, Ahn KY, Jung DS. Botulinum toxin type A treatment for contouring of the lower face. Dermatol Surg. 2003;29:477-8.

8. Brandt FS, Boker A. Botulinum toxin for rejuvenation of the neck. Clin Dermatol. 2003;21(6):513-20.

9. Levy PM. The "Nefertiti lift": a new technique for specific recontouring of the jawline. J Cosmet Laser Ther. 2007;9(4):249-52.

10. Tamura B. Toxina botulínica: concepção de beleza atual. 1st ed. São Paulo: Editora Santos; 2007.

11. Prado AS, Parada F, Andrades P, Fuentes P. Platysma chemical denervation with botox before neck lift. Plast Reconstr Surg. 2010;126(2):79e-81e.

12. Lowe NJ, Yamauchi P. Cosmetic uses of botulinum toxins for lower aspects of the face and neck. Clin Dermatol. 2004;22:18-22.

13. Carruthers A, Carruthers J, Monheit GD, Davis PG, Tardie G. Multicenter, randomized, parallel-group study of the safety and effectiveness of onabotulinumtoxinA and hyaluronic acid dermal fillers $(24-\mathrm{mg} / \mathrm{ml}$ smooth, cohesive gel) alone and in combination for lower facial rejuvenation. Dermatol Surg. 2010;36 Suppl 4:2121-34.

14. •• Raspaldo H, Niforos FR, Gassia V, et al. Lower-face and neck antiaging treatment and prevention using onabotulinumtoxin $\mathrm{A}$ : the 2010 multidisciplinary French consensus-part 1. J Cosmet Dermatol. 2011;10(2):131-4. This article shows that as a result on sophisticated treatment approaches, more specific targeted injections, and better understanding of lower facial and neck aging, more satisfying therapeutic results can be achieved for patients and clinicians.

15. • Rohrich RJ, Hanke CW, Busso M, et col. Facial soft-tissue fillers conference: assessing the state of the science. Plast Reconstr Surg. 2011 Apr;127(4 Suppl):22S-S. Erratum in: Plast Reconstr Surg. 2011 May;127(5):2138-40. The American Society of Plastic Surgeons and the American Academy of Dermatology established a panel of leading experts in the field of soft-tissue fillers to examine and discuss issues of patient safety, efficacy, and effectiveness in relation to the approved and off-label use of soft-tissue fillers and other factors, including the training and level of experience of individuals administering fillers.

16. - Sattler G, Carruthers A, Carruthers J, et al. Validated assessment scale for neck volume. Dermatol Surg. 2012;38(2 Spec No):343-5. This article explains that sagging of the neck aesthetic area is an important indicator of age.

17. - Narins RS, Carruthers J, Flynn TC, et al. Validated assessment scales for the lower face. Dermatol Surg. 2012;38(2 Spec No):333-4. This article shows that aging in the lower face leads 
to lines, wrinkles, depression of the corners of the mouth, and changes in lip volume and lip shape, with increased sagging of the skin of the jawline.

18. - Goldberg DJ. With this filler/volumizing agent now becoming available, the interest in non-surgical facial sculpturing will continue to expand. J Cosmet Laser Ther. 2008;10(3):133. This article shows that interest in non-surgical facial sculpturing will continue to expand.

19. - Pilsl U, Anderhuber F. The chin and adjacent fat compartments. Dermatol Surg. 2010;36(2):214-62. The chin compartment is well demarcated from adjacent compartments. The limits of the submental compartment vary. The jowl compartment is clearly demarcated from adjacent compartments.

20. • Hatef DA, Koshy JC, Sandoval SE, Echo AP, Izaddoost SA, Hollier LH. The submental fat compartment of the neck. Semin Plast Surg. 2009;23(4):288-91. The anatomic understanding of the superficial compartments of the head and neck are evolving. Recently, studies have shown that the superficial fat is sequestered into separate "compartments"; however, the superficial anatomy of the submental region of the neck has yet to be defined, and improved understanding of this area may lead to advances in our ability to rejuvenate the neck. 